\title{
Beitrag zur Paracelsus-Ikonographie
}

Von Walter Hugelshofer, Zürich

\section{Sehr verehrter Herr Professor Milt!}

Sie legen mir Heft 3/4 aus dem Jahrgang 1951 der Vierteljahresschrift «Gesnerus» vor und ersuchen um die Stellungnahme des Kunsthistorikers zu einem darin von dem seither verstorbenen Luzerner Augenarzte Dr. J. Strebel mitgeteilten neuen Bildnis nach Paracelsus.

Der Wunsch, von einem so außergewöhnlichen Menschen wie Paracelsus Bildnisse zu kennen, die erlauben würden, daraus einen neuen Zugang zu ihm zu gewinnen, ist verständlich genug. Es geht dabei aber ähnlich wie bei ZwINGLI, den er in Einsiedeln nur knapp verfehlt hat: erst als man nach dem frühen und unerwarteten Tode der vollen Bedeutung des Verstorbenen recht bewußt wurde, regte sich das Bedürfnis, ein Bildnis zu haben. Dem von Dr. StRebel veröffentlichten Stich kommt leider keinerlei authentischer Aussagewert zu. Wohl behauptet die mitgestochene Legende, der Dargestellte sei Paracelsus. Er ist aber einer 1684 in Nymwegen in Holland erschienenen Schrift entnommen und weist auch stichtechnisch (Kreuzlagen!) die Eigenheiten eines Reproduktionsstiches des 17.Jahrhunderts auf. Er ist nicht, wie Dr. Strebel annahm, eine Arbeit von Wenzel Hollar, dessen zahlreiche Stiche genau katalogisiert worden sind, sondern eine ausdrucksarme anonyme Kopie nach Hollars Stich, der im Verzeichnis Partheys unter Nummer 1543 aufgeführt worden ist und der seinerseits eine im Louvre in Paris verwahrte Silberstiftzeichnung HoLBEINs mit dem Datum 1520 nach einem unbekannten, noch fast im Knabenalter stehenden jungen Mann reproduziert. Den Stich Hollars findet man abgebildet unten rechts auf Seite 196 des von Professor PAUL Ganz 1911 redigierten Holbein-Bandes der Klassiker der Kunst. In der Anmerkung dazu wird auf die Zeichnung im Louvre verwiesen. Derselbe Paul Ganz hat sie in seiner monumentalen Publikation der Handzeichnungen des jüngeren HaNs Holbein auf Tafel XXXIII/3 reproduziert. Auch wenn die Legende es anders sagt, ist also nicht Paracelsus, sondern ein unbekannter junger Mann dargestellt.

Was nun aber die irreführende Aufschrift betrifft, hat man ihr ebensoviel Glauben zu schenken wie anderen Legenden dieser Art aus dem wundergläubigen 17. Jahrhundert, die kühnlich die Bildnisse Wilhelm Telds, 
Rudolfs von Habsburg oder Hans Waldmanns vorführen und damit dem allzeit wachen Bilderhunger antworten. Sie stehen in einer Reihe mit den Legenden unserer «Illustrierten» von heute, die auch nicht immer mit Sicherheit den Erwartungen und Ansprüchen der auf rasche Publizität drängenden Beschauer gerecht zu werden vermögen. Die Herausgeber des Nijmwegener Bandes besaßen vermutlich kein authentisches ParacelsusBildnis, das ihren Wünschen entsprach, und so erfanden sie eben eines. Es sieht nicht so aus, als ob die Aussichten, heute ein noch unbekanntes Paracelsus-Bildnis zu finden, viel größer geworden wären.

Ich bin mit meinem besten Gruß Ihr

\section{Walter Hugelshofer}

Nachschrift: Diese Klarstellung eines Paracelsus betreffenden ikonographischen Problems schien besonders wünschenswert. Der plötzliche Tod des Autors der hier besprochenen Arbeit ließ es schicklich erscheinen, dieselbe etwas zurückzustellen. Wir freuen uns aber, sie gerade in dieses Heft aufnehmen zu können.

B. MrLt 